\title{
Article \\ Accurate Electron Drift Mobility Measurements in Moderately Dense Helium Gas at Several Temperatures
}

\author{
Armando Francesco Borghesani
}

check for updates

Citation: Borghesani, A.F. Accurate Electron Drift Mobility

Measurements in Moderately Dense Helium Gas at Several Temperatures. Atoms 2021, 9, 52. https://doi.org/ 10.3390 /atoms 9030052

Academic Editor: Grzegorz Piotr Karwasz

Received: 14 July 2021

Accepted: 2 August 2021

Published: 4 August 2021

Publisher's Note: MDPI stays neutral with regard to jurisdictional claims in published maps and institutional affiliations.

Copyright: (c) 2021 by the authors. Licensee MDPI, Basel, Switzerland. This article is an open access article distributed under the terms and conditions of the Creative Commons Attribution (CC BY) license (https:// creativecommons.org/licenses/by/ $4.0 /)$.
Dipartimento di Fisica \& Astronomia "Galileo Galilei” and CNISM, University of Padua, I-35131 Padua, Italy; armandofrancesco.borghesani@unipd.it; Tel.: +39-049-827-7019

\begin{abstract}
We report new accurate measurements of the drift mobility $\mu$ of quasifree electrons in moderately dense helium gas in the temperature range $26 \mathrm{~K} \leq T \leq 300 \mathrm{~K}$ for densities lower than those at which states of electrons localized in bubbles appear. By heuristically including multiple-scattering effects into classical kinetic formulas, as previously done for neon and argon, an excellent description of the field $E$, density $N$, and temperature $T$ dependence of $\mu$ is obtained. Moreover, the experimental evidence suggests that the strong decrease of the zero-field densitynormalized mobility $\mu_{0} N$ with increasing $N$ from the low up to intermediate density regime is mainly due to weak localization of electrons caused by the intrinsic disorder of the system, whereas the further decrease of $\mu_{0} N$ for even larger $N$ is due to electron self-trapping in cavities. We suggest that a distinction between weakly localized and electron bubble states can be done by inspecting the behavior of $\mu_{0} N$ as a function of $N$ at intermediate densities.
\end{abstract}

Keywords: electron mobility; multiple-scattering effects; disordered systems; weak localization

\section{Introduction}

The study of the transport properties of excess electrons in dielectric gases or liquids may shape detailed knowledge of the dynamics and energetics of electron states in disordered media and of the relationship between the electron-host atom interactions and the thermodynamic properties of the system. In particular, the negative density effect, i.e., the decline of the electron drift mobility $\mu$ with increasing gas number density $N$, initially observed in dense helium [1-7], has attracted a great deal of theoretical work [8-15] because of the possible connection between multiple-scattering effects at a high density and the electron localization induced by the intrinsic disorder of the medium $[16,17]$.

Several multiple-scattering theories have been developed for the thermal electrons mobility $\mu_{0}$, i.e., in the limit of vanishingly small density-reduced electric field $E / N \rightarrow 0$, where $E$ is the drift electric field. All these theories are based on a complex shift of the electron kinetic energy in a dense medium [18,19] and on quantum corrections to the electron-atom scattering rate when the electron mean free path $\ell$ and the thermal electron wavelength $\lambda_{T}$ become comparable [11,20]. They are proved quite successful at the description of the density dependence of the zero-field limit of the density-normalized electron mobility $\mu_{0} N$ in helium.

Unfortunately, the theoretical prediction for $\mu_{0} N$ in dense neon was proven wrong or, at best, incomplete [21], mainly because it was based on the assumption of a nearly energy-independent momentum-transfer scattering cross section $\sigma_{\mathrm{mt}}$. Moreover, these theories explained the different density effects (negative in helium and neon but positive in argon, i.e., $\mu_{0} N$ increasing with $N[22,23]$ ) by invoking different physical mechanisms according to the sign of the scattering length $a$.

In order to give a unique description of the electron-atom scattering process in a dense gas, independent of the sign of $a$, we have developed a model [24] that heuristically incorporates the multiple-scattering effects introduced by previous theories $[25,26]$. 
We briefly recall here the main three multiple-scattering effects that are taken into account in the heuristic model. The first one is the density-dependent energy shift $E_{k}(N)$ of the electron kinetic energy $\epsilon . E_{K}$ is the zero-point kinetic energy arising from the exclusion of the electron from the hard-core volume of the atoms [27]. It can explicitly be obtained by replacing the fluid structure with a locally ordered array of hard-sphere scatterers and by matching the electron wave function with its asymptotic expression at the surface of the Wigner-Seitz sphere [28] of volume $4 \pi r_{\mathrm{ws}}^{3} / 3=1 / N$ centered on each atom, thereby getting [29]

$$
E_{k}(N)=\frac{\hbar^{2}}{2 m} k_{0}^{2}
$$

$m$ is the electron mass, $\hbar=h / 2 \pi$, and $h$ is the Planck's constant. The wave vector $k_{0}$ is determined by the eigenvalue equation

$$
\tan \left[k_{0} r_{\mathrm{ws}}+\eta_{0}\left(k_{0}\right)\right]=k_{0} r_{\mathrm{ws}}
$$

in which $\eta_{0}(k)$ is the $s$-wave phaseshift [30]. In order to account for the superposition of the tails of the atomic potentials, $-\eta_{0}\left(k_{0}\right) / k_{0}$ is replaced by the hard-core radius of the HartreeFock potential $\tilde{a}=\sqrt{\sigma_{T}\left(k_{0}\right) / 4 \pi}$, in which $\sigma_{T}$ is the total scattering cross section [27]. This energy shift produces a large effect if the momentum-transfer scattering cross section is a rapidly varying function of the electron energy.

The second effect is due to correlations among scatterers. The electron wave packet encompasses a volume of the gas whose linear dimension is of the order of the electron wavelength $\lambda=\hbar / \sqrt{2 m \epsilon}$. This volume contains many atoms, and the electron is scattered off all of them simultaneously. The total amplitude of the scattered wave is obtained by summing up coherently all partial scattering amplitudes contributed by each atom. The net result is that the scattering cross section is weighted by the static structure factor of the gas $S(0)=N k_{\mathrm{B}} T \chi_{T}$ [31]. Here, $k_{\mathrm{B}}$ is the Boltzmann's constant, and $\chi_{T}$ is the gas isothermal compressibility.

Finally, the third multiple-scattering effect is the enhancement of the electron backscattering rate due to quantum self-interference of the electron wave function scattered off atoms located along paths connected by time-reversal symmetry [32]. The strength of this effect depends on the ratio of the electron thermal wavelength $\lambda_{T}$ to the electron mean free path $\ell=1 / N \sigma_{\mathrm{mt}}$. For $\lambda_{T} / \ell \leq 1$, a perturbative treatment is adequate yielding for the scattering rate $v$ the linearized expression [10]

$$
v(\epsilon)=v_{0}\left(1+f \frac{\lambda}{\ell}\right)=v_{0}\left(1+f \frac{\hbar v_{0}}{\epsilon}\right),
$$

in which $v_{0}=\sqrt{2 \epsilon / m} N \sigma_{\mathrm{mt}}$ is the scattering rate in the dilute gas limit, and $f$ is a number of order unity [10]. This perturbative treatment is adequate for argon [23] and neon [21] because their cross sections are relatively small at thermal and shifted energies.

However, for helium, $\sigma_{\mathrm{mt}}$ is so large and the experimental $N$ so high that $\lambda / \ell \gtrsim 1$. In this case, we are in presence of the so-called weak localization regime in the jargon of the theory of disordered systems $[33,34]$. If disorder is enhanced by increasing $N$, electrons become completely localized with exponentially decaying wave functions (known also as Anderson localized states) [16], and a mobility edge appears at the finite energy $\epsilon_{c}[35,36]$.

At the mobility edge the scattering rate diverges. Polischuk obtains the mobility edge with a sophisticated diagrammatic technique [12]. The same result can be obtained by following a more simple, intuitive approach. The correction term enhancing the scattering rate in Equation (3) must be proportional to the actual scattering rate $v(\epsilon)$ rather than to the unperturbed scattering rate $v_{0}$. It is then easy to get

$$
v(\epsilon)=\frac{v_{0}}{1-f \hbar v_{0} / 2 \epsilon}=\frac{v_{0}}{1-f \lambda / \ell}
$$


that perfectly agrees with the result of Polischuk [12]. The location of the mobility edge corresponds to the Ioffe-Regel criterion for localization $\lambda \simeq \ell$ [25], and the mobility edge energy is obtained as

$$
\epsilon_{c}=\frac{2}{m}\left[\frac{f}{2} \hbar N S(0) \sigma_{\mathrm{mt}}\left(\epsilon_{c}\right)\right]^{2} .
$$

in which the cross section enhancement due to correlation among scatterers is included. We note that there is some disagreement in the literature about the value of $f$. Its value is $f=2 \pi / 3 \approx 2.09$ for Polischuk [12] and $f=2$ for Atrazhev [10]. The difference is quite small $(\approx 4 \%)$ and does not significantly affect the results.

The heuristic model [23] is obtained by including the above-mentioned multiplescattering effects into the equation for the mobility of the classical kinetic theory [26]. Its advantage is that it also predicts the electric field dependence of $\mu$ in addition to the $N$ dependence of $\mu_{0}$. Moreover, it does not entail adjustable parameters and can be applied to all noble gases independently of the sign of the electron-atom scattering length.

The density-normalized mobility is given by [26]

$$
\mu N=-\frac{e}{3}\left(\frac{2}{m}\right)^{1 / 2} \int_{\epsilon_{c}}^{\infty}\left[\frac{\epsilon}{\sigma_{\mathrm{mt}}^{\star}(\epsilon)}\right] \frac{\mathrm{d} g(\epsilon)}{\mathrm{d} \epsilon} \mathrm{d} \epsilon .
$$

$g(\epsilon)$ is the Davydov-Pidduck energy distribution function [26,37]

$$
g(\epsilon)=A \exp \left\{-\int_{0}^{\epsilon}\left[k_{\mathrm{B}} T+\frac{M e^{2}}{6 m z}\left(\frac{E}{N \sigma_{\mathrm{mt}}^{\star}(z)}\right)^{2}\right]^{-1} \mathrm{~d} z\right\}
$$

Here, $M$ is the atom mass, and $A$ is the normalization constant given by enforcing the condition $\int_{0}^{\infty} \epsilon^{1 / 2} g(\epsilon) \mathrm{d} \epsilon=1 . \sigma_{\mathrm{mt}}^{\star}$ is the effective momentum-transfer scattering cross section that takes into account the three multiple-scattering effects, and it is expressed by

$$
\sigma_{\mathrm{mt}}^{\star}(\epsilon)=\mathcal{F}(w) \sigma_{\mathrm{mt}}(w)\left[1-f \hbar \frac{\mathcal{F}(w) \sigma_{\mathrm{mt}}(w)}{(2 m w)^{1 / 2}}\right]^{-1},
$$

in which $w=\epsilon+E_{k}$ is the shifted energy. $\mathcal{F}(w)$ is given by

$$
\mathcal{F}(w)=\frac{1}{4 w^{2}} \int_{0}^{2 w} q^{3} S(q) \mathrm{d} q .
$$

For not too large values of the exchanged momentum $q$, the Ornstein-Zernike approximation [38] can be used, yielding a Lorentzian form of the structure factor

$$
S(q)=\frac{S(0)+(q L)^{2}}{1+(q L)^{2}}
$$

in which $L^{2}=0.1 l^{2}[S(0)-1]$, and $l \approx 0.1 \mathrm{~nm}$ is the short-range correlation length [39].

The previous formulas do not have any adjustable parameters and allow the researchers to compute $\mu N$ as a function of $E, N$, and $T$ for any gas whose cross sections and thermodynamic equation of state are known. In particular, the density dependence of $\mu_{0} N$ can simply be obtained by setting $E / N=0$ in Equation (7).

The model we have outlined is developed to describe the mobility of quasifree electrons by possibly taking into account the existence of weakly localized, non-propagating states. It has proven extremely successful when applied to argon [23,40] and neon $[21,24]$.

However, in this latter case, sufficiently high-density and low-temperature electrons get self-trapped in (partially) empty cavities giving birth to low-mobility electron bubbles for which the use of the heuristic model is no longer applicable. In cold, dense helium 
gas, the formation of electron bubbles is also a well-known phenomenon [1-3,6,7,41,42]. The comparison of the theoretical predictions for the quasifree electrons has, thus, to be made only for such $N$ and $T$ ranges, in which electron bubbles are not significantly present. It is interesting to note, however, that the previous multiple-scattering theories for $\mu_{0} N$ were applied to an extended $N$ range, thereby leading, in our opinion, to some confusion about the relationship between Anderson-localized and bubble states.

Therefore, we have carried out new, accurate measurements in broad $T$ and $N$ ranges in order to gain insight both in the process of electron bubble formation at high densities and at higher temperatures and in the behavior of quasifree electrons up to intermediate densities. The results on bubble formation have already been published [42]. In this paper, we will present mobility data from low up to intermediate density in the range $26 \mathrm{~K} \leq T \leq 300 \mathrm{~K}$ in order to investigate the behavior of quasifree electrons, compare the previous multiple-scattering theories with the present heuristic model, and shed some light on the relationship between disorder-induced non-propagating states and self-trapped states in bubbles.

The paper is organized as follows: In Section 2, the details of the experiments are briefly described. In Section 3, the experimental results are presented and discussed. In Section 4, we will discuss if experimental data allow the distinction between Anderson-localized states and electron bubbles with the aid of the prediction of the heuristic model.

Following the Conclusions in Section 5, in Appendix A, we will give some details on the different effects on the mobility of the two relevant energy scales, $E_{K}$ and $\epsilon_{c}$, which are necessary for the description of the experimental electron mobility.

\section{Experimental Details}

The measurements are carried out using the pulsed photoemission technique and apparatus exploited in previous measurements of electron mobility, $\mathrm{O}_{2}^{-}$mobility, and resonant electron attachment in dense helium, neon, and argon gases and have already been thoroughly described $[21,40,42-47]$. We recall here only the main technical features of the experiment.

A high-pressure cell is mounted on the cold head of a cryocooler inside a triple-shield thermostat. The cell can withstand pressure up to $P \approx 10 \mathrm{MPa}$ and can be cooled down to $T \approx 25 \mathrm{~K}$. The cell temperature is stabilized within $\pm 0.01 \mathrm{~K}$, and the pressure is measured with an accuracy of $\pm 1 \mathrm{kPa}$. The gas used is ultra-high purity helium with nominal $\mathrm{O}_{2}$ content of $1 \mathrm{ppm}$. However, in order to make accurate mobility measurements, the impurity content must be lowered by recirculating the gas in a closed loop through a $\mathrm{LN}_{2}$-cooled activated-charcoal trap and a commercial Oxisorb cartridge [48]. The final impurity content is estimated to be a fraction of one ppb. The gas density $N$ is computed from the measured values of $T$ and $P$ by means of an accurate equation of state $[49,50]$.

The parallel plate drift capacitor is located in the cell and is energized by a d.c. highvoltage generator [51]. A thin slice of electrons is photoinjected from the cathode by a short pulse $(\approx 4 \mu \mathrm{s})$ of VUV light produced by a Xe flashlamp [52] and is drifted towards the anode. The injected charge amounts to 4 through $400 \mathrm{fC}$, depending on the gas pressure in the cell and on the applied electric field strength, and is low enough to avoid spacecharge effects.

The current induced at the anode by the drifting electrons is passively integrated to improve the signal-to-noise ratio. The voltage signal is acquired by a digital oscilloscope and fetched by a personal computer for offline analysis. Several signals are acquired for any experimental conditions and are software-averaged to improve the signal quality.

The drift time $\tau$ is obtained by analyzing the time evolution of the voltage signal at the anode [53]. The drift velocity is obtained as $v_{D}=\tau / d$, where $d$ is the drift distance, and the mobility is obtained as $\mu=v_{D} / E$, where $E$ is the applied electric field. The estimated accuracy on $\mu$ is much better than $5 \%$. 


\section{Experimental Results and Discussion}

In this section, the experimental data will be presented and discussed.

\subsection{The Need for Accurate Measurements}

The present measurements in helium are aimed at validating the heuristic model developed for the description of the mobility of quasifree electrons in dense noble gases [23]. This goal justifies the need for new accurate measurements of the mobility. Actually, the main attention in the past was focused onto the negative density effect shown by $\mu_{0} N$ that eventually leads to the formation of electron bubbles. As $\mu_{0} N$ decreases by nearly five orders of magnitude in a restricted density range at low temperature [2-4,6,7,41], the mobility at low and medium density, where no localization takes yet place, was not investigated with the necessary accuracy in the past. In order to clarify this point, the present $\mu_{0} N$ data for $T=77.2 \mathrm{~K}$ are compared in Figure 1, with one of the most accurate experiments at nearly the same temperature [4].

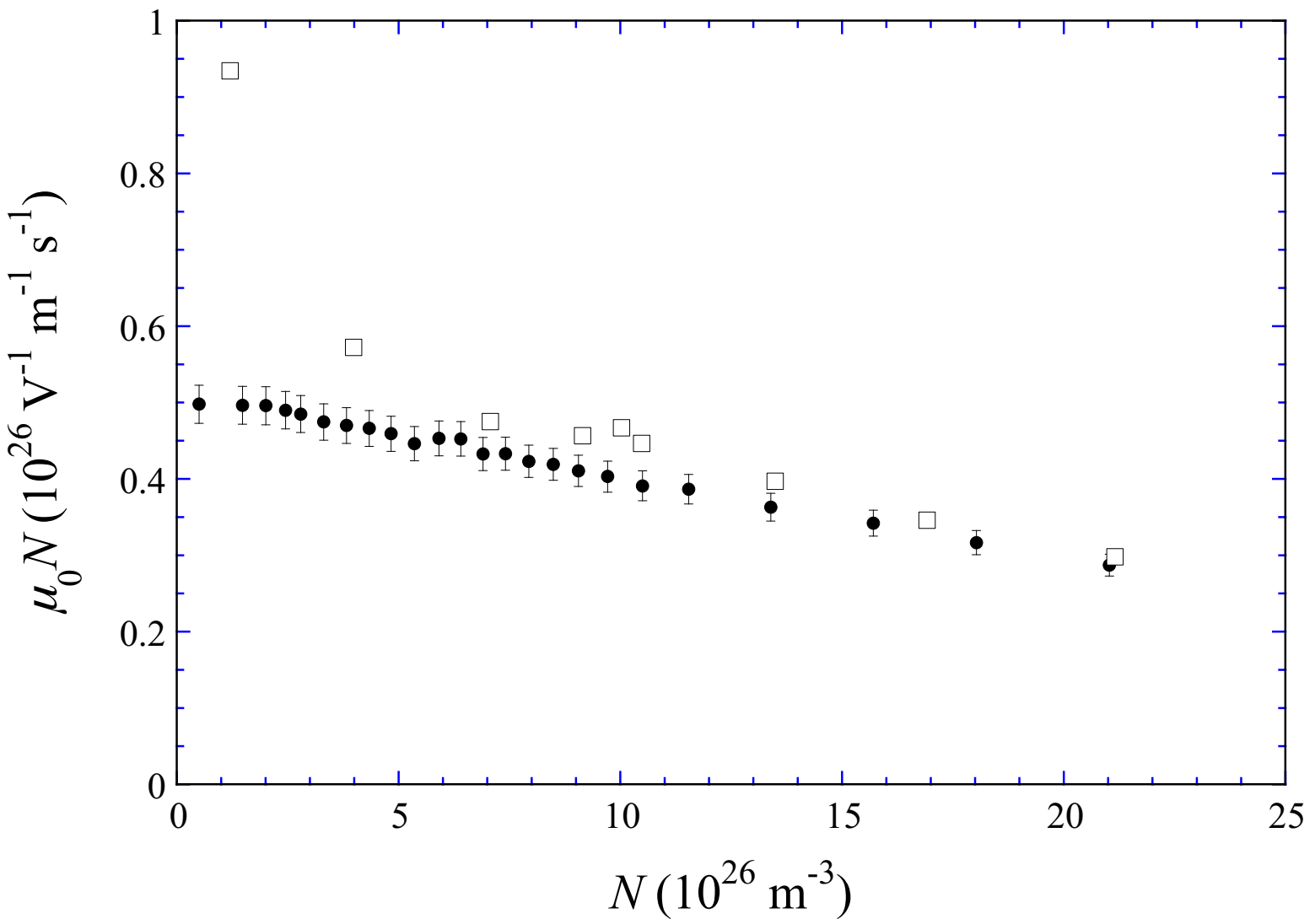

Figure 1. $\mu_{0} N$ vs. $N$ at $T \approx 77 \mathrm{~K}$. Comparison of the present measurements accuracy at $T=77.2 \mathrm{~K}$ (closed points) with literature data at $T=77.6 \mathrm{~K}$ (open squares) [4].

Literature data are more scattered than the present ones, especially at low density, where they strongly disagree with the classical kinetic theory prediction based on the commonly accepted momentum-transfer scattering cross section [30]. On the contrary, it will be shown in the following that the present data agree very well with this prediction. At lower temperatures, the accuracy of literature data in the low density range is even worse $[2,3,41]$.

\subsection{Choice of the Correct Density Range to Explore}

Another important point to be discussed before proceeding is the determination of the density range in which the quasifree electron mobility is not affected by the presence of electrons localized in cavities. As the mobility of the electron bubbles is roughly four to 
five orders of magnitude smaller than that of quasifree electrons, any significant presence of them would spoil the comparison of the experimental outcome with the theory for quasifree electrons.

Actually, the present measurements were carried out in very broad $N$ and $T$ ranges because of the great interest in ascertaining whether the phenomenon of electron self-trapping in cavities also occurs at higher temperatures than previously observed. The high-density $\mu_{0} N$ data [42] have shown that localization takes place at any $T$, indeed, provided that $N$ is large enough to yield an excess Helmoltz free energy of the localized state with respect to the quasifree one $\Delta \mathcal{A}(N, T) \lesssim 0$ with $|\Delta \mathcal{A}(N, T)| \gtrsim k_{\mathrm{B}} T$, in which $k_{\mathrm{B}}$ is the Boltzmann constant. The excess free energy $\Delta \mathcal{A}(N, T)$ was computed by adopting a simple electron bubble model [54], taking into account that the gas has no surface tension and that the helium atoms have non-negligible thermal energy at the temperatures of the experiment. The threshold density $N^{\star}$ at which quasifree electrons and electron bubbles are equiprobable is obtained by solving the equation

$$
\Delta \mathcal{A}\left(N^{\star}, T\right)=0,
$$

yielding a quite satisfactory agreement with the data [42]. However, at $N^{\star}$, the fraction of electron bubble states is $50 \%$, and its contribution to the mobility is far from negligible. Thus, the threshold density, above which they cannot actually be neglected any longer, must experimentally be determined by inspecting the electric field dependence of $\mu$ for different $N$. A typical example is shown in Figure 2 for $T=26.1 \mathrm{~K}$.

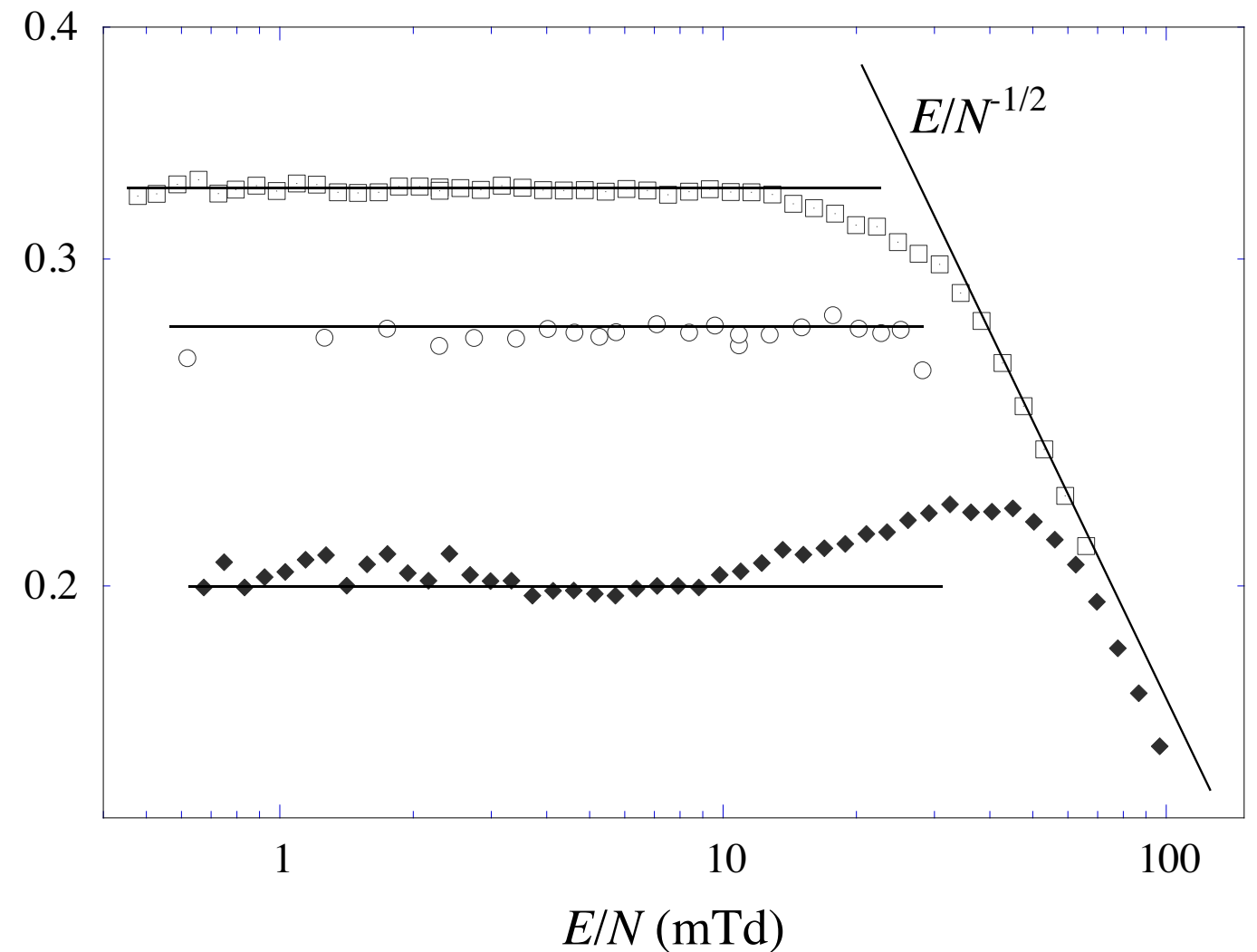

Figure 2. $\mu N$ vs. $E / N$ at $T=26.1 \mathrm{~K}$ for densities close to the onset of the electron self-trapping in bubbles. From top: $N=17.33,19.47$, and 22.76 in units of $10^{26} \mathrm{~m}^{-3} .1 \mathrm{mTd}=10^{-24} \mathrm{Vm}^{2}$. The line $\propto(E / N)^{-1 / 2}$ is the prediction of the classical kinetic theory for scattering of quasifree, epithermal electrons off hard spheres [26]. The presence of electron bubble states for the highest $N$ is proven by the rise of $\mu N$ above its zero-field limit (shown as a constant line) before joining the high-field behavior. 
Helium can be considered a hard-sphere scatterer to a good approximation as its momentum-transfer scattering cross section $\sigma_{\mathrm{mt}}$ is roughly independent of energy (it varies from $4.87 \AA^{2}$ at energy $\epsilon=0$ to $6.8 \AA^{2}$ at $\epsilon=1 \mathrm{eV}$ ) [30]. For such a system, the classical kinetic theory predicts that, at constant $T, \mu N$ is constant at weak electric field and is proportional to $(E / N)^{-1 / 2}$ at a high field. By inspecting Figure 2, in which the electric field dependence of $\mu N$ is shown for $T=26.1 \mathrm{~K}$ for some $N$, we note that $\mu N$ at the lower $N$ follows the classically expected behavior. If $N$ is further increased, the behavior of $\mu N$ changes even qualitatively. At weak fields, $\mu N$ is constant but, upon a further increase of the field strength, $\mu N$ increases with $E / N$ and shows a maximum before joining the density-independent, high-field region. This specific behavior, observed for $N$ large enough even at very low temperature [6,7], was previously reported also in dense neon gas [44] and is intepreted as the field-assisted hindrance to self-trapping or field-assisted release of electrons from the bubbles [55]. This interpretation is also supported by experimental evidence in liquid neon [56].

The logical consequence drawn from the observed field dependence of $\mu N$ is that a temperature-dependent threshold $N_{\text {th }}$ density exists for electron bubble states of low mobility to be experimentally detected. In Figure $3, N_{\text {th }}$ is reported along with the computed density $N^{\star}$.

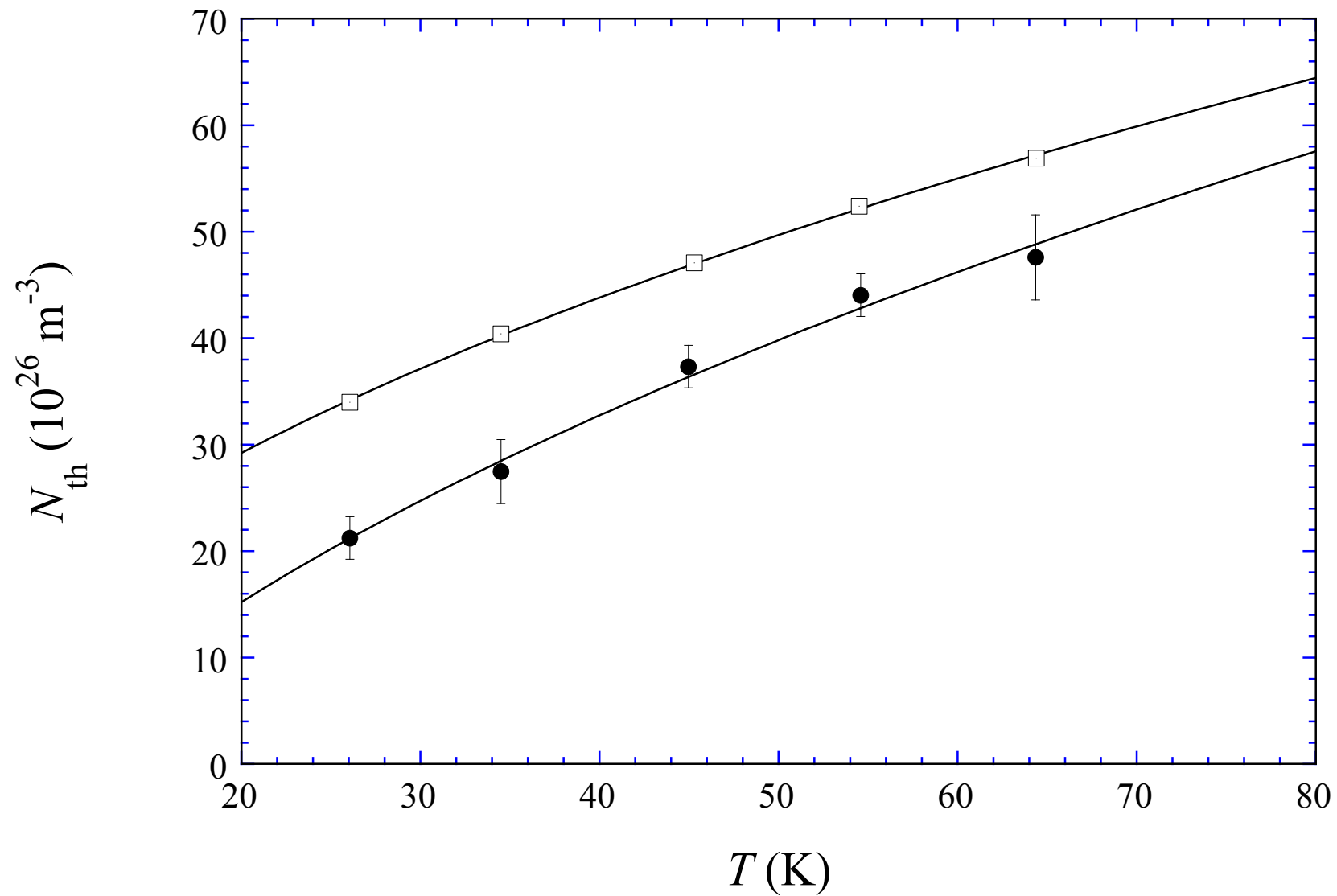

Figure 3. Temperature dependence of the threshold density $N_{\text {th }}$ for the onset of bubble state formation as determined by the inspection of the field dependence of $\mu N$ (closed points). Open squares: temperature dependence of the density $N^{\star}$ at which quasifree electrons and electron bubbles are equiprobable according to the bubble model [42]. The lines are only a guide for the eye.

As a result, the analysis of the mobility of quasifree electrons will be restricted to $N \leq N_{\text {th }}(T)$. 


\subsection{Density Dependence of the Zero-Field Limit of the Density Normalized Mobility}

In Figure $4, \mu_{0} N$ is shown as a function of $N$ for several $T$. As previously discussed, the data are shown only for $N<N_{\text {th }}$ to be sure that only quasifree electrons contribute to the mobility. At the highest temperatures, the pressure necessary to reach $N_{\text {th }}$ exceeds the experimental cell capability, and thus, the measurements are restricted to not too high density values. At all temperatures, $\mu_{0} N$ shows the well-known negative density effect that is extremely well described by the heuristic model, represented by the solid lines through the data in the figure.

According to the model, three density-dependent processes combined to determine the behavior of the mobility: the quantum density-dependent shift of the kinetic energy of the electron $E_{k}(N)$, the correlation among scatterers, and the quantum self-interference of the electron wave packet scattered by atoms located along paths connected by timereversal symmetry. In the case of helium, the first one is not very effective because $\sigma_{\mathrm{mt}}$ does not depend very much on the electron energy. On the contrary, this effect is very important in neon [21,43] and argon [22,24,40,57-59], whose cross sections are strongly energy dependent $[13,60]$.

The correlation among scatterers is a second process that increases the scattering cross section by the long-wavelegth limit of the static structure factor $S(0)=N k_{\mathrm{B}} T \chi_{T}$. The effect of $S(0)$ is very important close to the critical point, which, for helium, occurs for $T_{c}=5.2 \mathrm{~K}$ and $N_{c}=104.8 \times 10^{26} \mathrm{~m}^{-3}[49,50]$. As the present experiment is carried out for $T \gg T_{\mathcal{C}}, S(0) \approx 1$ always, its effect is quite negligible.

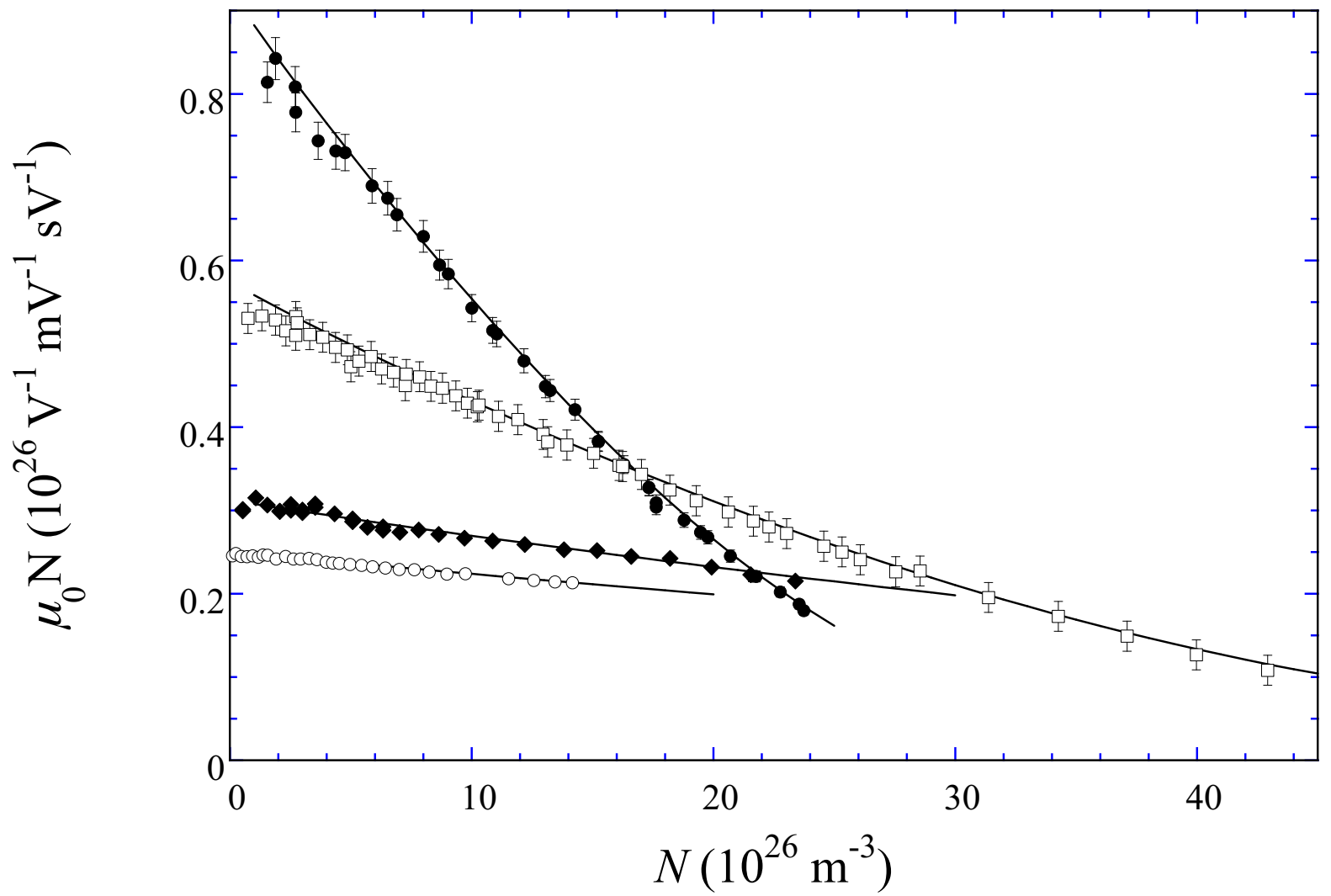

Figure 4. $\mu_{0} N$ vs. $N . T=26.1 \mathrm{~K}$ (closed points), $T=64.4 \mathrm{~K}$ (open squares), $T=199.5 \mathrm{~K}$ (closed diamonds), and $T=295.5 \mathrm{~K}$ (open points). Lines: heuristic model prediction. The data are only presented for $N \leq N_{\text {th }}$, at which electron bubbles are still either absent or present in a negligible proportion. The error bars for the highest $T$ are of the same size of the dots and are not shown.

The last process is the quantum self-interference of the electron wave packet scattered off atoms located along paths connected by time-reversal symmetry. As discussed in the 
Introduction, this process leads to an increase of the scattering rate, which is $\alpha \lambda / \ell$, i.e., proportional to the ratio of the electron quantum wavelength $\lambda$ to its mean free path $\ell$. However, if the scattering cross section is large, i.e., if the mean free path is short, and the temperature is low, i.e., the electron thermal wavelength is large, the quantum selfinterference process is so strong to lead to the weak electron localization via the appearance of the mobility edge $[11,12,17]$. Electrons in the low-energy tail of the distribution function below the mobility edge energy $\epsilon_{\mathcal{c}}$ do not propagate. In helium, this is the dominant process that produces most of the observed negative density effect of the mobility.

The mobility edge introduces an infrared cutoff in the electron energy distribution function, which gives rise to a strong exponential decrease in $\mu_{0} N$ with increasing $N$. On one hand, the quite fair agreement with experiments of the older theories, all of which are based on a complex shift of the electron energy in a dense disordered medium due to multiple-scattering effects [13-15], is explained by various additional assumptions (not all fully correct) invoked by the authors, as discussed in the literature $[8,9,20]$. Their apparent success is due to the fact that they predict an exponential decrease of the mobility with increasing $N$. It has also to be noted that these theories invoke different phenomena to explain the different density effects observed in repulsive gases (such as helium and neon) and in attractive gases (such as argon) $[10,12,14]$.

On the other hand, the heuristic model takes into account all the three multiplescattering effects in a natural way. Their relative weight is automatically accounted for by the strength and energy dependence of the scattering cross section and by the thermodynamic state of the gas through which electrons are drifted. As a further benefit, the heuristic model treats the scattering of low energy electrons in noble gases in a unified way independently of the sign of the scattering length.

The heuristic model shares with the theories of Atrazhev [10] and of Polischuk [12] the concept of enhancement of the scattering rate due quantum self-interference (that eventually leads to the appearance of the mobility edge) but accounts for the two additional multiple-scattering effects (quantum density-dependent shift of the electron kinetic energy in the dense disordered medium and correlation among scatterers), although their influence only marginally affects the electron mobility in helium in the particular thermodynamic conditions of the experiment.

\subsection{Validation of the Accuracy of the Present Experiment and of Its Outcome}

A way to validate the accuracy of the results of the present experiment is to show that the present data agree well with the prediction of the classical kinetic theory in the limit of low density [26]. If $N \rightarrow 0$, all multiple-scattering effects vanish. Both the mobility edge $\epsilon_{c}$ and the energy shift $E_{k}(N)$ tend to 0 and the long wavelength limit of the structure factor $S(0) \rightarrow 1$. In this limit, the classical formula predicts

$$
\mu_{0} N \rightarrow\left(\mu_{0} N\right)_{0}=\frac{4 e}{3 \sqrt{2 \pi m\left(k_{\mathrm{B}} T\right)^{5}}} \int_{0}^{\infty} \frac{\epsilon}{\sigma_{\mathrm{mt}}(\epsilon)} \mathrm{e}^{-\epsilon / k_{\mathrm{B}} T} \mathrm{~d} \epsilon .
$$

The zero-density extrapolation of the mobility obtained from the investigated isotherms (some of which are reported in Figure 4) are compared in Figure 5 with the theoretical prediction, Equation (12), based on O'Malley's low-energy electron-helium cross section [30]. The data are in excellent agreement with the classical prediction, thereby lending credibility to the accuracy of the experiment. It has to be noted that both the data as well as the theoretical line are extremely well fitted to an inverse power law of the temperature, namely $\left(\mu_{0} N\right)_{0} \propto T^{-0.536}$. The exponent differs from the value $-1 / 2$, which is typical of gas of hard-sphere scatterers, because of the weak energy dependence of the electron-helium momentum-transfer scattering cross section. 


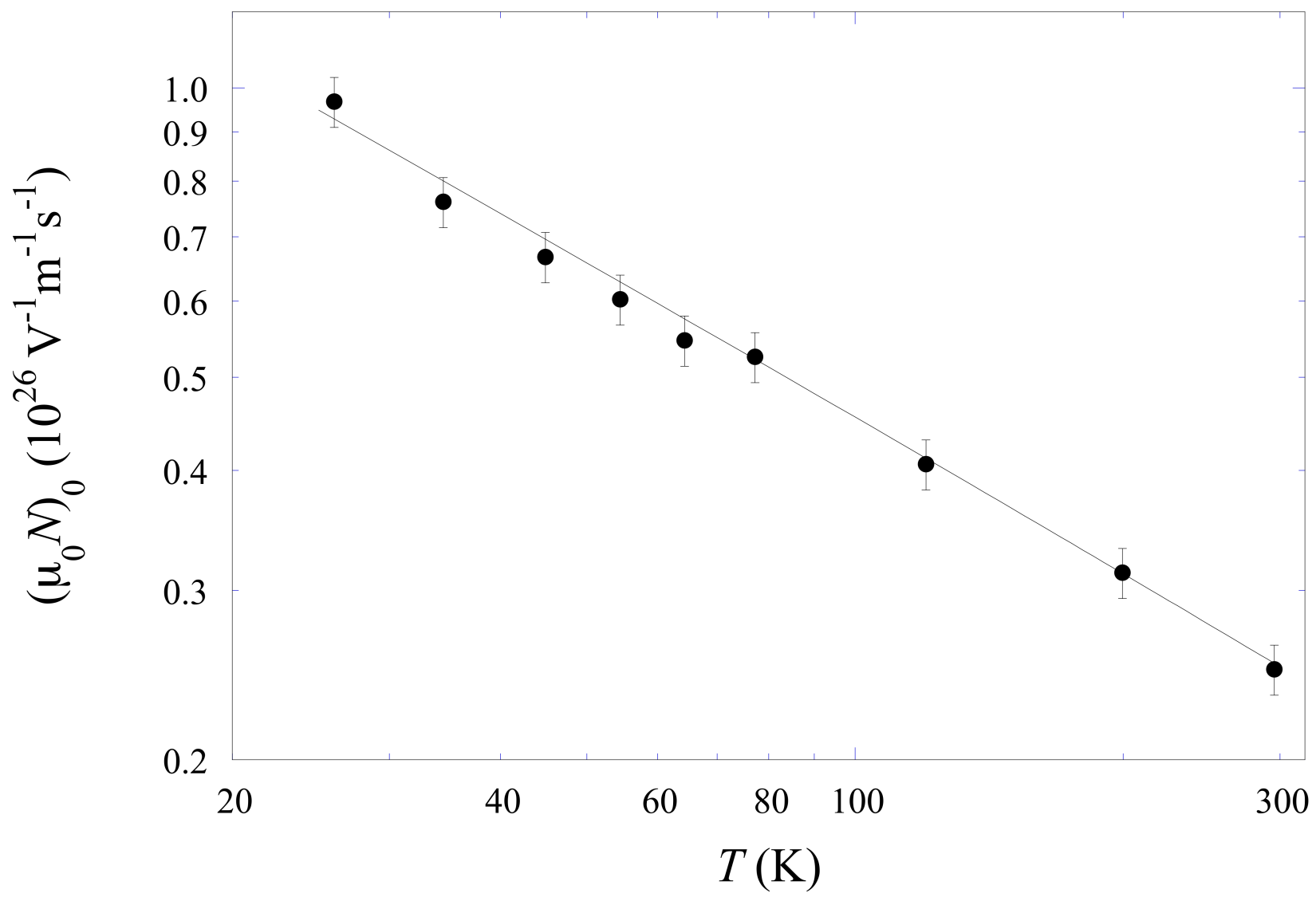

Figure 5. $\left(\mu_{0} N\right)_{0}$ vs. T. The solid line is the prediction of the heuristic model which, for $N \rightarrow 0$, must coincide with the classical kinetic prediction [26]. Data and theory are well fitted to the inverse power law $T^{-0.536}$ close to the $T^{-1 / 2}$ behavior of a gas of hard-sphere scatterers.

An additional confirmation of the validity of the heuristic model is obtained by inspecting how it is able to reproduce the electric field dependence of the experimental data. In Figure 6, we show typical $\mu N$ vs. $E / N$ data for $T=64.4 \mathrm{~K}$ for several $N<N_{\text {th }}$, i.e., for densities below the onset of electron bubble formation. The heuristic model is able to accurately describe the field dependence of $\mu N$ for well over a decade in $N$ and nearly three decades in $E / N$. Similar results are obtained at all temperatures.

A small discrepancy between the experiment and model can still be spotted in the transition region between thermal and epi-thermal behavior. On one hand, this discrepancy could be ascribed to imperfect knowledge of the scattering cross section at energies higher than thermal. On the other hand, it has to be noted that, for any given $N$, the same energy shift $E_{K}(N)$ is used for all fields and energies in Equation (6), although it is obtained by a solving Equation (2), which is only valid for $s$-wave scattering. 


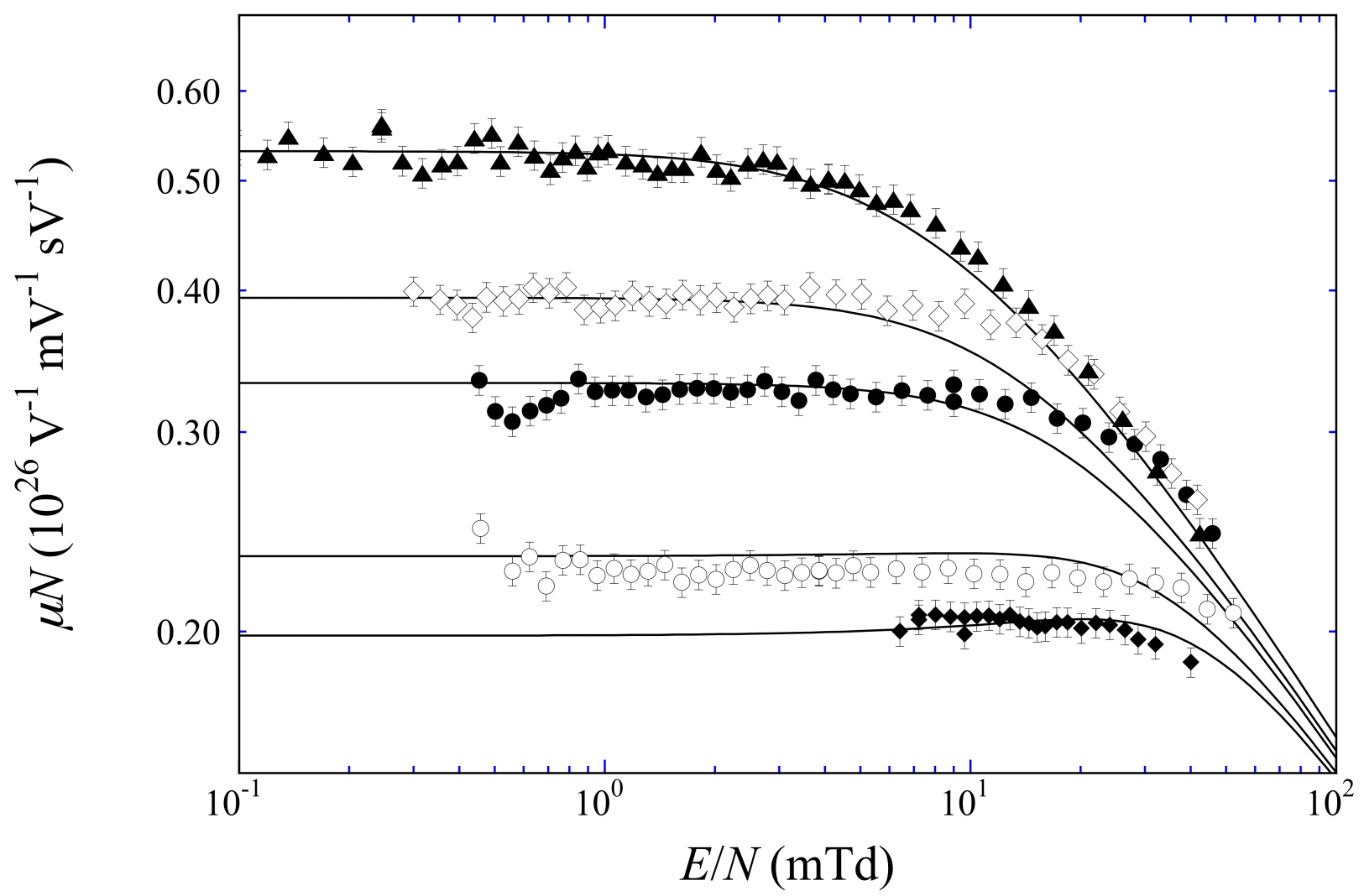

Figure 6. $\mu N$ vs. $E / N$ for $T=64.4 \mathrm{~K}$. Densities (from top, in units of $10^{26} \mathrm{~m}^{-3}$ ): $N=2.79,12.95,18.20,27.54,31.38$. The solid lines are the predictions of the heuristic model.

\section{Weakly Localized States vs. Electron Bubble States}

It is well known that electrons in cold dense helium, either liquid or gas, do give origin to states localized in cavities (for a review, see Ref. [61]). The cavities form as a consequence of the delicate balance between the free energies of the quasifree and the localized electrons and because the medium is compliant enough not to withstand the quantum pressure exerted by the wavefunction of the localized electron that pushes away the nearby atoms, thereby digging out the cavity.

On the other hand, it is also well known that a static structural disorder can lead to the vanishing of electron diffusion because of the formation of Anderson-localized states $[12,16,17,35]$. In this case, the electron wave function exponentially decays with distance owing to multiple-scattering effects induced by the disorder. Under this respect, helium is a school case of a dense, disordered system consisting of (nearly) hardsphere scatterers.

It still unclear, however, if Anderson-localized states might be precursors of electron bubbles. Actually, non-propagating states might remain for a time long enough to favor the enhancement and stabilization of the cavity because of the medium compliance.

We believe that the actual measurements might give some hints to solve this issue. As an argument, we will investigate the experimental behavior of $\mu N$ as a function of $E / N$ for densities at which electron bubbles definitely exist [42].

In Figure 7, the field dependence of $\mu N$ is reported for $T=26.1 \mathrm{~K}$ and for $N=32.36 \times 10^{26} \mathrm{~m}^{-3}$ and $N=34.31 \times 10^{26} \mathrm{~m}^{-3} . \mu N$ is constant at weak fields and shows a rapid enhancement towards the electron epithermal behavior $\sim E / N^{-1 / 2}$ for stronger fields. 


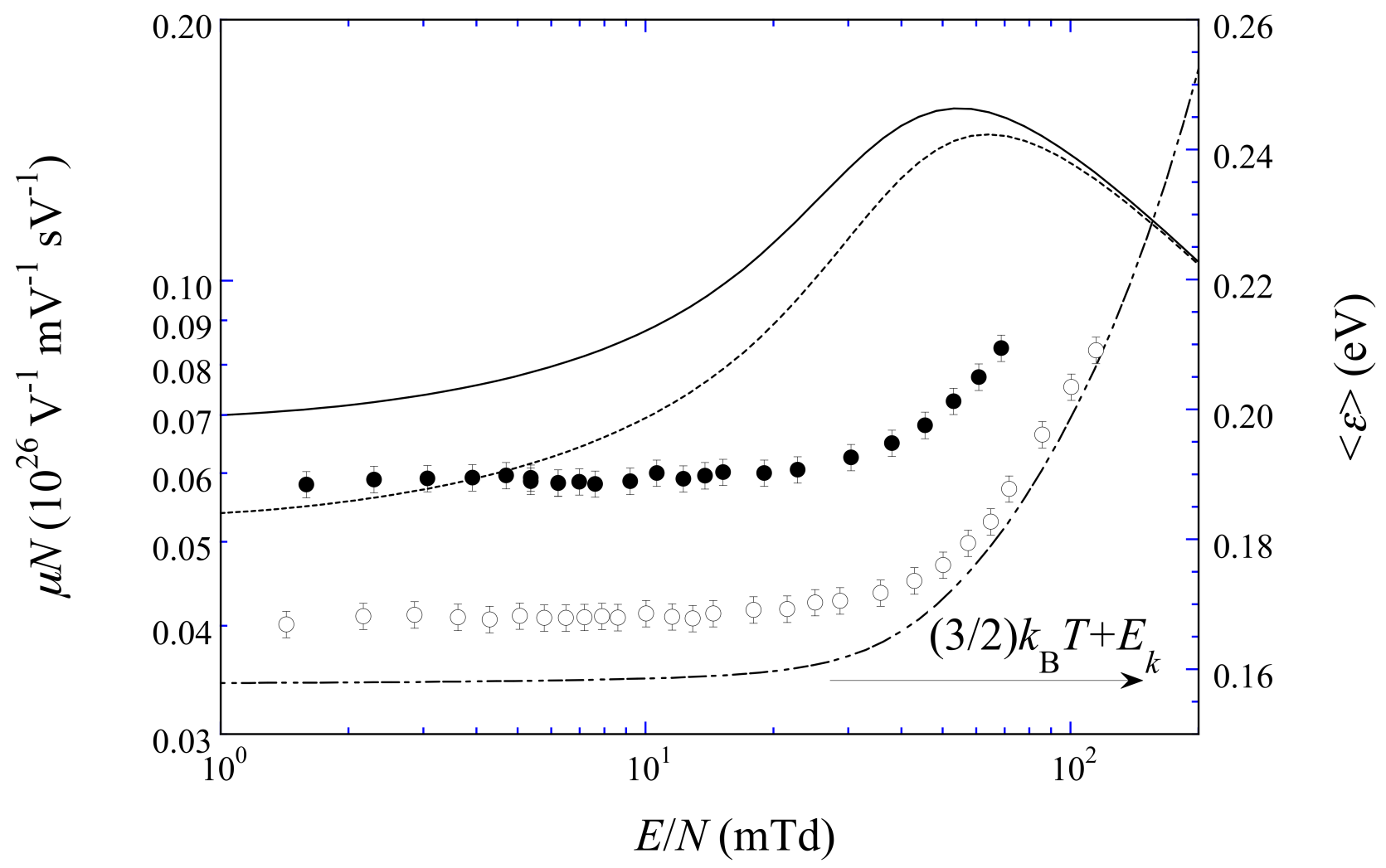

Figure 7. Left scale: $\mu N$ vs. $E / N$ for $T=26.1 \mathrm{~K}$ for $N=32.36$ (closed points) and $N=34.31$ (open points). The solid and dotted lines are the predictions of the heuristic model. $N$ is in units of $10^{26} \mathrm{~m}^{-3}$. Right scale: the average electron energy for $N=34.31 \times 10^{26} \mathrm{~m}^{-3}$ (dash-dotted line), which includes the contribution of $E_{k}(N)$.

For these densities, the heuristic model fails at reproducing the low-field mobility predicting far too large values. The rationale for this failure might be that, for these densities, there is coexistence of both weakly localized electrons and self-trapped electrons in bubbles. The heuristic model obviously accounts for the Anderson-localized states through the infrared cutoff in the electron energy distribution function due to the mobility edge. On the contrary, the measured mobility is a weighted sum of the contributions of the quasifree states, which are very mobile, and of the electron bubbles, which, though very slowly, do still propagate.

The heuristic model predicts that $\mu N$ increases upon increasing $E / N$ before reaching the epithermal behavior. This increase takes place at much weaker $E / N$ values than experimentally observed. The transition between the low- and high-field behavior experimentally occurs in the same field region in which the average electron energy (dashed-dotted line in Figure 7) starts increasing above its thermal value $\langle\epsilon\rangle \geq(3 / 2) k_{\mathrm{B}} T+E_{k}(N)$. We note that, in this field range, the electron drift velocity becomes comparable with the sound speed $\approx 300 \mathrm{~m} / \mathrm{s}[50,62]$.

We, thus, draw the conclusion that Anderson-localized states cannot be precursors of electron bubbles. Actually, if they were such precursors, the decrease of their number upon increasing field should lead to an increase of the measured mobility that, by contrast, remains constant. The mobility only increases at stronger fields where the average electron energy and the drift velocity are large enough to hinder the electron self-trapping process in cavities.

Such point of view is further confirmed, in our opinion, if the fraction of quasifree electrons is compared with the mobility behavior as a function of the reduced field. 
The fraction $f_{\text {free }}$ of quasifree states with energy in excess of the mobility edge energy $\epsilon_{c}$ is given by

$$
f_{\text {free }}=\int_{\epsilon_{c}}^{\infty} \epsilon^{1 / 2} g(\epsilon) \mathrm{d} \epsilon .
$$

In Figure 8, we plot the experimental mobility (closed points) and the model prediction (solid line) for $T=34.5 \mathrm{~K}$ and $N=41.74 \times 10^{26} \mathrm{~m}^{-3}$ and compare their behavior with $f_{\text {free }}$ (dashed line, right scale).

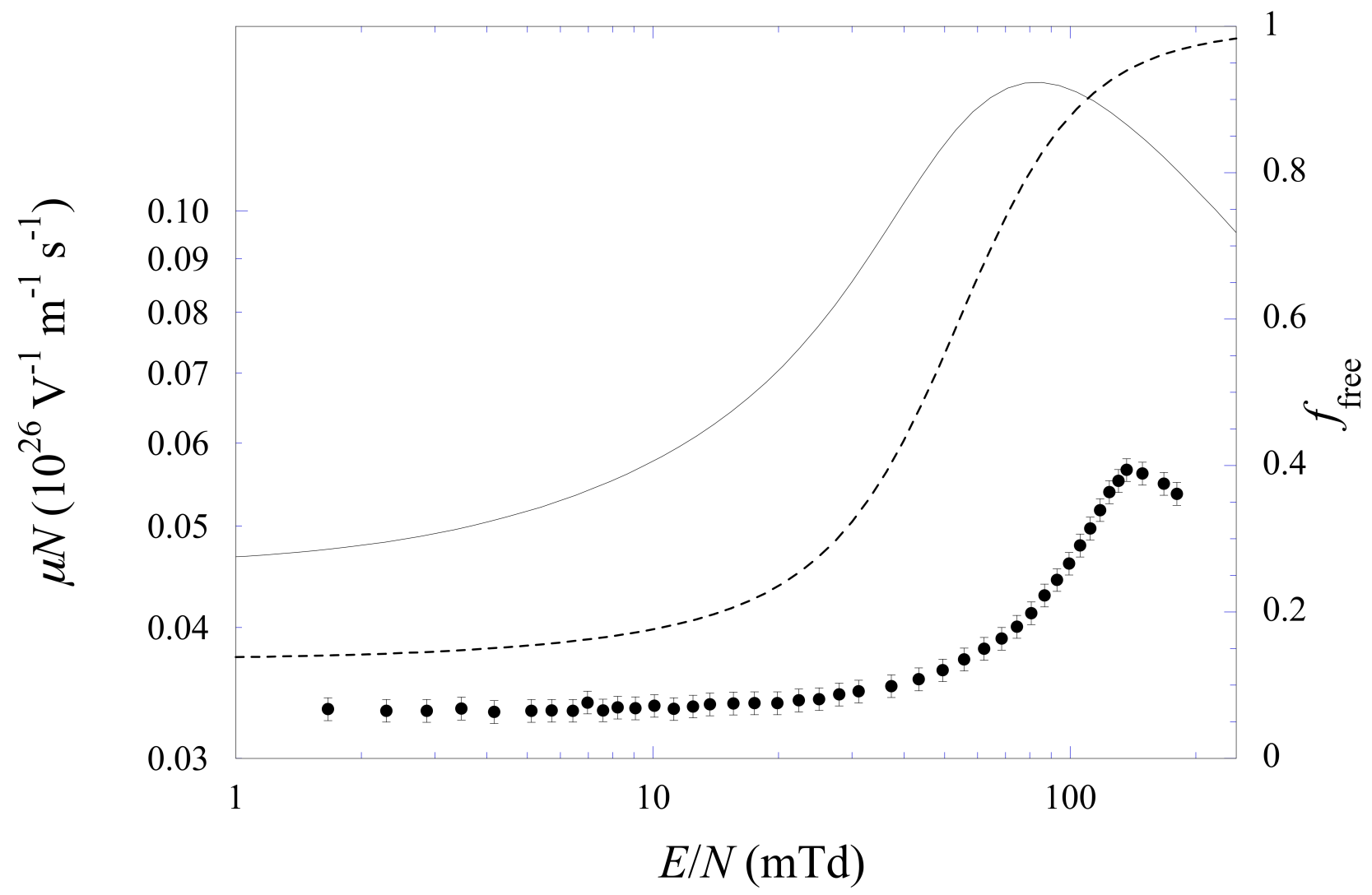

Figure 8. Left scale: $\mu N$ vs. $E / N$ for $T=34.5 \mathrm{~K}$ for $N=41.74 \times 10^{26} \mathrm{~m}^{-3}$ (closed points) and prediction of the heuristic model (solid line). Dashed line: the fraction of quasifree electrons $f_{\text {free }}$ (right scale).

At such high a density, $\mu_{0} N$ is well below the predicted value by the heuristic model and by all other theoretical models $[10,12-14]$ because a non-negligible fraction of electrons are localized in bubbles and significantly contributes to the mobility decline with density. By inspecting Figure 8 , it can be noted that the quasifree electron fraction is $f_{\text {free }} \approx 10 \%$ at low fields and rapidly increases towards $f_{\text {free }}=100 \%$ at high fields. However, its rise begins at much weaker field strength than the mobility rise. Therefore, we can conclude that the reduction of the fraction of non-propagating states below the mobility edge is not the principal mechanism of the mobility increase with electric field at high densities and that these weakly localized states are probably not the precursors of the electron states self-trapped in cavities.

\section{Conclusions}

In this paper, we have presented new and accurate experimental measurements of electron mobility in helium gas in a wide temperature range for low to intermediate densities. The experimental ranges have been selected in a way to exclude the presence of electron bubbles that might spoil the theoretical analysis. 
We have shown that the heuristic model, originally developed for neon and argon, also works very well for helium. On one hand, it encompasses the multiple-scattering effects taken into account also by previous theories. It supersedes them because all the multiple-scattering effects are included at once in a unified picture. In particular, for helium, the main effect is produced by the presence of a disorder-induced mobility-edge that leads to a nearly exponential decrease of the zero-field limit of the density-normalized mobility $\mu_{0} N$ by shrinking the phase space available to freely propagating electrons.

On the other hand, at least for the noble gases, the heuristic model treats the electronatom scattering process in a dense, disordered medium as a unique phenomenon that does not depend if the electron-atom interaction potential is mainly attractive or repulsive. The different density effect shown by different gases is only a manifestation of the energy dependence of the scattering cross sections.

Moreover, in comparison with the previous theories, the present heuristic model is also able to describe the electric field dependence of the quasifree electron mobility with great accuracy.

In helium, owing to the near energy independence of the scattering cross section and to the distance from the criticality of the present measurements, the most important multiple-scattering process affecting the electron mobility is the existence of a disorderproduced mobility edge at which the scattering cross section diverges. States with energy below the mobility-edge energy do not propagate and are weakly-localized, and thus, the phase space of propagating electrons shrink. The resulting infrared cutoff in the electron energy distribution function leads to a strong, nearly exponential decrease in $\mu_{0} N$ with increasing density that explains the approximate success of previous theories.

The availability of an accurate theoretical model and experimental measurements has allowed us to suggest a clear distinction between disorder-induced, non-propagating, Anderson-localized states and electron bubbles. From the theoretical analysis of the experimental data in a thermodynamic state in which electron bubbles are present, the conclusion can be drawn that Anderson-localized states may coexist with electron bubbles.

It would be interesting if an analysis similar to the present one could be carried out on measurements of electron mobility in dense carbon dioxide [63,64], ammonia [65], and methanol [66] that show a significant negative density effect and even self-trapping in cavities.

Funding: This research received no external funding.

Institutional Review Board Statement: Not applicable.

Informed Consent Statement: Not applicable.

Data Availability Statement: The data presented in this study are only available on request from the corresponding author because they are still under consideration for further analysis.

Acknowledgments: The author acknowledges useful discussions with M. Santini , A.M. De Riva, D. Neri, and P. Lamp. Thanks are due to G. Delfitto for technical assistance.

Conflicts of Interest: The author declares no conflict of interest.

\section{Appendix A. Influence of $E_{k}(N)$ and of $\epsilon_{c}$ on the Mobility of Quasifree Electrons}

Two characteristic energies have to be considered in the heuristic model to produce an accurate description of the mobility data without any adjustable parameters, namely the density-dependent energy shift $E_{k}(N)$ and the mobility edge energy $\epsilon_{\mathcal{c}} . \epsilon_{\mathcal{c}}$ slightly depends on $T$ because of $S(0)$. However, for all $T$ and $N$ of the present experiment, $S(0) \approx 1$ within less than $10 \%$, and $\epsilon_{\mathcal{C}}$ turns out to practically be temperature independent.

The two energies are very different values, as shown in Figure A1. Roughly speaking, $E_{k}$ is more than $\approx 20$ times larger than $\epsilon_{\mathcal{c}}$ at all densities. 


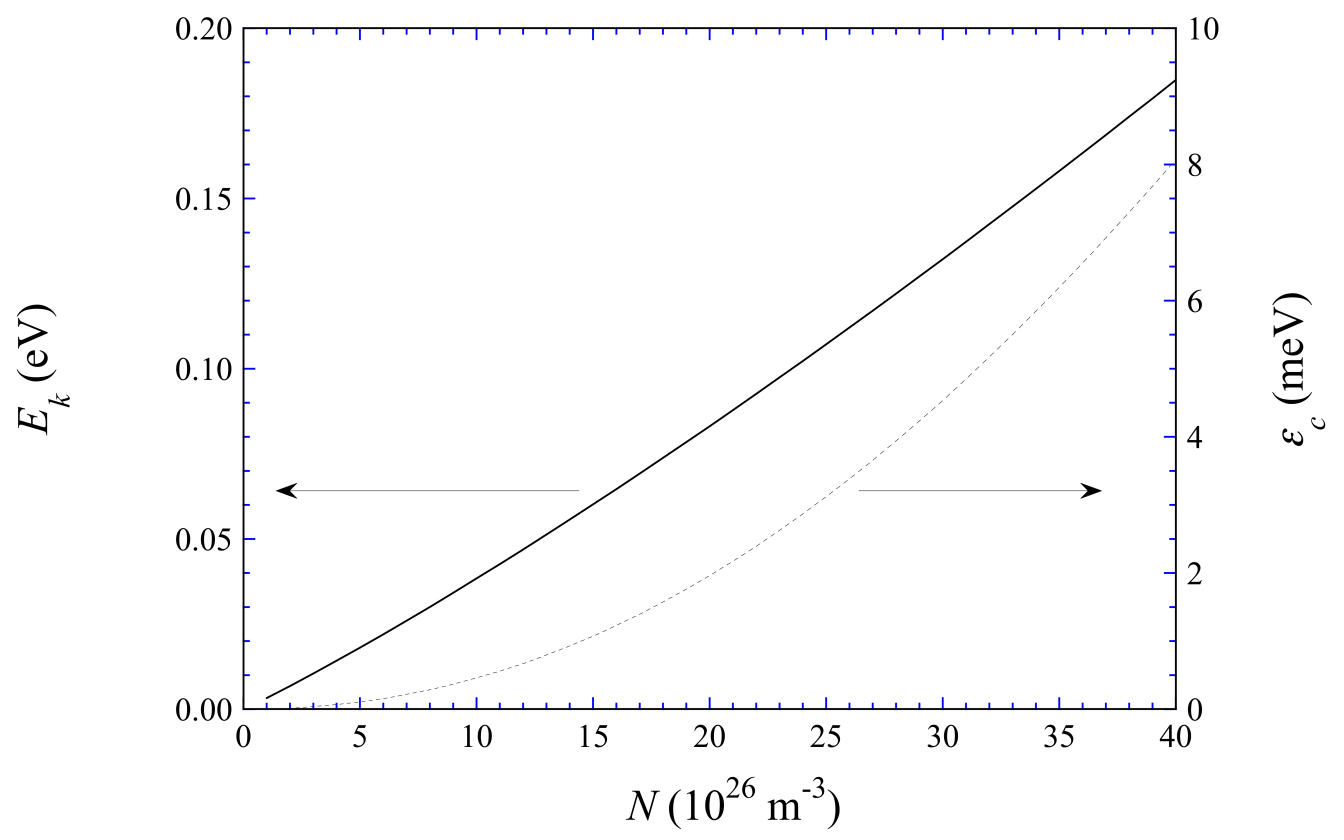

Figure A1. The density dependence of the energy shift $E_{k}$ (left scale) and of the mobility edge energy $\epsilon_{\mathcal{C}}$ (right scale).

Despite this big difference between the two quantities, in the case of helium gas, the smaller one produces the bigger effect on the mobility. Actually, $E_{k}(N)$ produces a rigid shift of the electron energy distribution function whose zero is shifted from $\epsilon=0$ to $\epsilon=E_{k}(N)$. By a suitable change of variables in the integrals for the mobility [23], it can be shown that the effect of $E_{k}$ is to force the evaluation of the energy-dependent electron-atom scattering cross section at the shifted energy, as demonstrated by Equation (8). If the cross section is nearly independent of energy, as is the case of helium, its evaluation at a shifted energy gives nearly the same value, and the effect on the mobility is small. On the contrary, the action of $E_{k}$ produces the dominant effect in neon [21,43,44] and argon [22,23,40,58], whose cross sections very rapidly vary with energy.

The action of the mobility edge energy is more subtle. It introduces an infrared cutoff in the mobility integrals. Electron states with energies below $\epsilon_{\mathcal{C}}$ do not propagate. Thus, the cutoff actually shrinks the phase space available to the freely propagating states. For thermal electrons and (nearly) energy-independent cross sections, the zero-field density normalized mobility is given by

$$
\mu_{0} N \propto \int_{\epsilon_{c}}^{\infty} \epsilon \mathrm{e}^{-\epsilon / k_{\mathrm{B}} T} \mathrm{~d} \epsilon=\mathrm{e}^{-\epsilon_{\mathcal{c}} / k_{\mathrm{B}} T} \int_{0}^{\infty}\left(z+\epsilon_{c}\right) \mathrm{e}^{-z / k_{\mathrm{B}} T} \mathrm{~d} z \sim \mathrm{e}^{-\epsilon_{\mathcal{c}} / k_{\mathrm{B}} T}\left(\mu_{0} N\right)_{0}
$$

Loosely speaking, the classical mobility $\left(\mu_{0} N\right)_{0}$ is thus multiplied by an exponential factor of the form $\exp \left(-\epsilon_{c} / k_{\mathrm{B}} T\right)$. Actually, the exact integral weakens the quadratic density dependence of $\epsilon_{c}$ in the argument of the exponential. In any case, the strong density dependence of the exponential leads to the observed negative density effect of the electron mobility in helium. Furthermore, in neon and argon, the quantum self-interference effect leads to the appearance of a mobility edge. However, in these gases, the cross section at thermal energies is so small that $\epsilon_{c} \approx 0$ and can safely be neglected, thus allowing the other multiple-scattering effects to fully manifest.

As mentioned before, the infrared cutoff $\epsilon_{c}$ leads to a reduction of the fraction $f_{\text {free }}$ of freely propagating electron states. It is interesting to investigate how $f_{\text {free }}$ depends on $T, N$, and $E / N$ as a consequence of the presence of $\epsilon_{c}$. 
In Figure A2, we show the influence of $N$ on the field dependence of $f_{\text {free }}$ at constant $T$. For all $N$, the action of the electric field is to broaden the electron energy distribution function so that the fraction of states with energy $\epsilon>\epsilon_{\mathcal{c}}$ always increases with the increasing $E / N$.

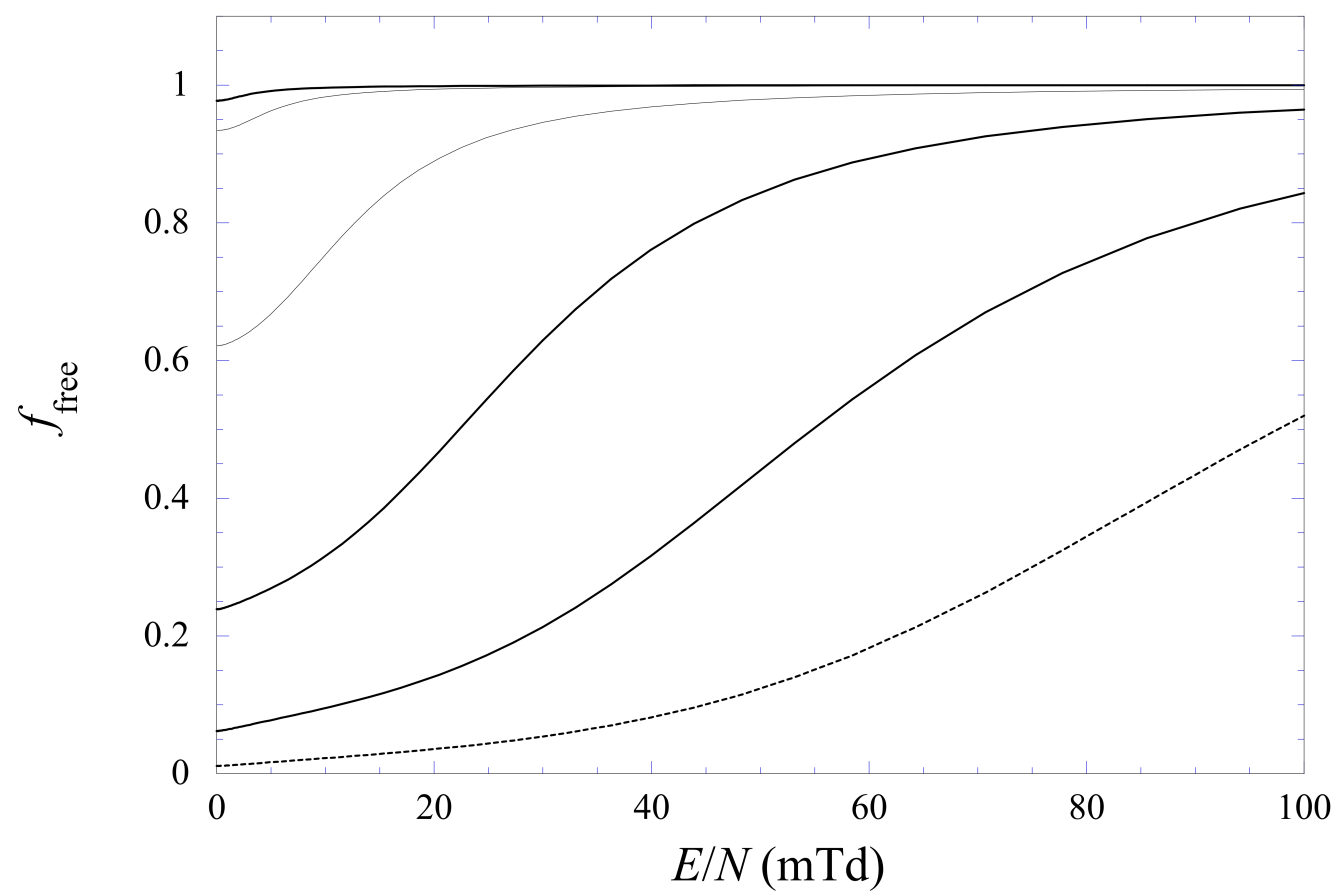

Figure A2. $f_{\text {free }}$ as a function of $E / N$ at $T=26.1 \mathrm{~K}$. From top: $N: 5,10,20,30,40,50$ (in units of $10^{26} \mathrm{~m}^{-3}$ ). Note that for $T=26.1 \mathrm{~K}$, the threshold density is $N_{\text {th }} \gtrsim 20$.

At a low density, $f_{\text {free }}$ is practically always equal to 1 . Upon increasing $N, f_{\text {free }}$ steadily decreases. The higher the density is, the larger the field strength required to produce larger proportions of quasifree electrons is.

Similar considerations can be made about the effect of temperature on $f_{\text {free }}$ at constant density, as shown in Figure A3.

At constant $N$ and $T$, the action of the field is the same as discussed for the previous figure. At constant $E / N$ and $N$, the action of $T$ is clear. Upon increasing $T$, the electron energy distribution gets broader, and the average electron energy increases, thereby increasing the fraction of electrons with energy in excess of $\epsilon_{c}$. It has to be once more noted that, at the density of Figure A3 for $T \lesssim 50 \mathrm{~K}$, a large number of electrons are self-trapped in bubbles.

It is finally interesting to note that fraction of quasifree states at $E / N=0$ is a universal function of $y_{c}=\sqrt{\epsilon_{c} / k_{\mathrm{B}} T} \propto N / \sqrt{T}$

$$
\lim _{E / N \rightarrow 0} f_{\text {free }}=\frac{2}{\sqrt{\pi}} y_{c} \mathrm{e}^{-y_{c}^{2}}+\operatorname{erfc}\left(y_{c}\right),
$$

in which $\operatorname{erfc}(x)$ is the error function [12]. 


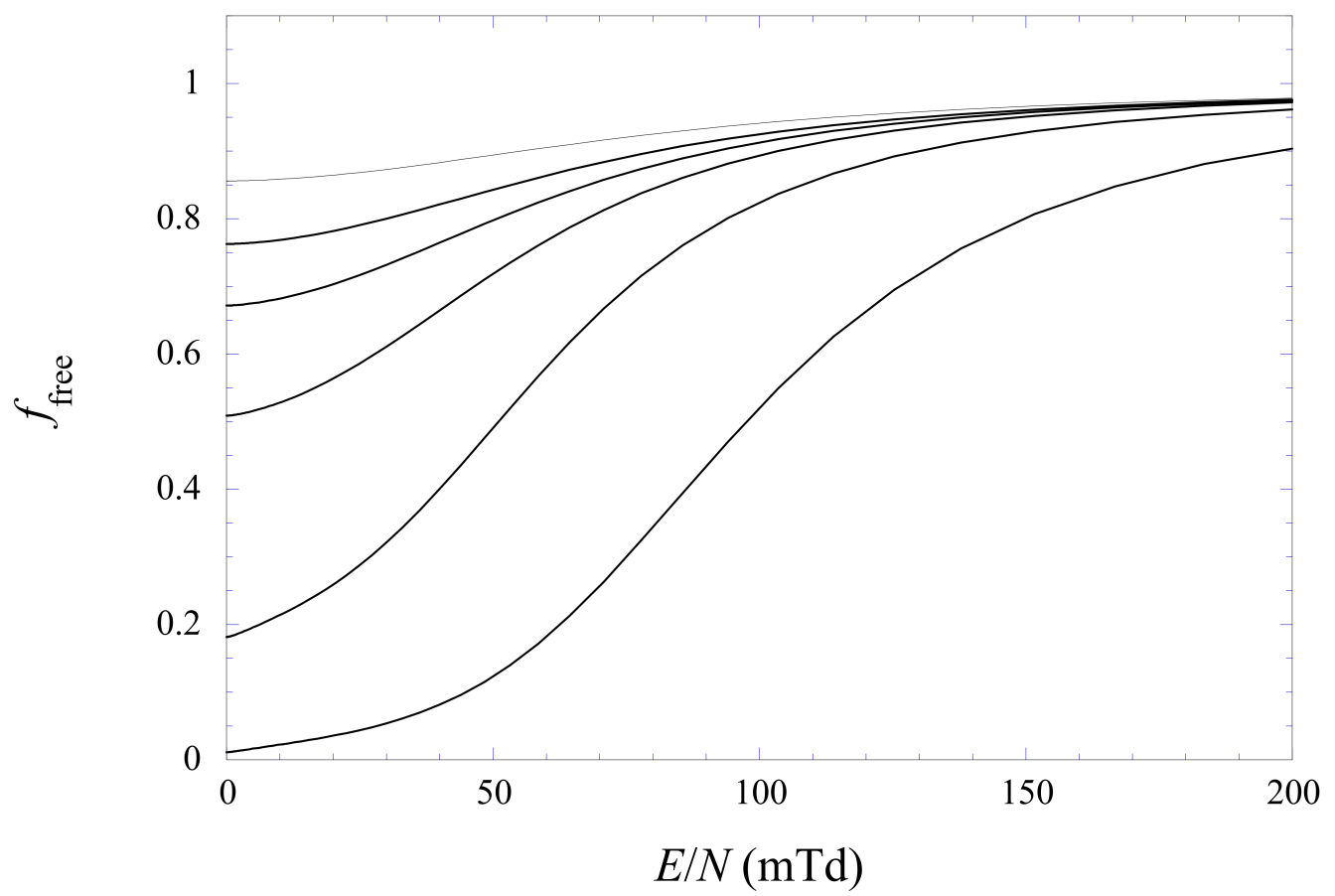

Figure A3. $f_{\text {free }}$ vs. $E / N$ at $N=50 \times 10^{26} \mathrm{~m}^{-3}$ for several $T$. From top: $T(K)=300,200,150,100,50,26.1$.

\section{References}

1. Levine, J.; Sanders, T.M. Anomalous electron mobility and complex negative ion formation in low-temperature helium vapor. Phys. Rev. Lett. 1962, 8, 159-161. [CrossRef]

2. Levine, J.L.; Sanders, T.M. Mobility of electrons in low-temperature helium gas. Phys. Rev. 1967, 154, 138-149. [CrossRef]

3. Harrison, H.R.; Sander, L.M.; Springett, B.E. Electron mobility and localization in dense $\mathrm{He}_{4}$ gas. J. Phys. B At. Mol. Phys. 1973, 6, 908-917. [CrossRef]

4. Bartels, A. Density dependence of electron drift velocities in helium and hydrogen at 77.6 K. Appl. Phys. 1975, 8, 59-64. [CrossRef]

5. Schwarz, K.W.; Prasad, B. Dynamics of electron localization in dense helium gas. Phys. Rev. Lett. 1976, 36, 878-881. [CrossRef]

6. Schwarz, K.W. Anomalous electron mobilities in dense helium gas. Phys. Rev. Lett. 1978, 41, 239-242. [CrossRef]

7. Schwarz, K.W. Electron localization in dense helium gas: New experimental results. Phys. Rev. B 1980, 21, 5125-5136. [CrossRef]

8. Iakubov, I.T.; Polischuk, A.Y. Influence of multiple scattering processes on the electron mobility in moderately dense gases. Phys. Lett. A 1982, 91, 67-69. [CrossRef]

9. Iakubov, I.T.; Polischuk, A.Y. Quantum density corrections to mobility and dispersion law for electrons in a medium of disordered scatterers. J. Phys. B At. Mol. Phys. 1982, 15, 4029-4041. [CrossRef]

10. Atrazhev, V.M.; Iakubov, I.T. The electron drift velocity in dense gases. J. Phys. D Appl. Phys. 1977, 10, 2155-2163. [CrossRef]

11. Ya Polischuk, A. Quantum corrections to electron conductivity in a disordered medium of anisotropic scatterers. J. Phys. B At. Mol. Phys. 1983, 16, 3853-3858. [CrossRef]

12. Polischuk, A.Y. Theory of electron mobility in dense gases with small polarizability. Phys. B+C 1984, 124, 91-95. [CrossRef]

13. O'Malley, T.F. Multiple scattering effect on electron mobilities in dense gases. J. Phys. B At. Mol. Phys. 1980, 13, 1491-1504. [CrossRef]

14. Braglia, G.L.; Dallacasa, V. Theory of electron mobility in dense gases. Phys. Rev. A 1982, 26, 902-914. [CrossRef]

15. O'Malley, T.F. General Model For Electron Drift and Diffusion in a Dense Gas. J. Phys. B At. Mol. Opt. Phys. 1992, 25, 163-180. [CrossRef]

16. Anderson, P.W. Absence of diffusion in certain random lattices. Phys. Rev. 1958, 109, 1492-1505. [CrossRef]

17. Cutler, M.; Mott, N.F. Observation of Anderson localization in an electron gas. Phys. Rev. 1969, 181, 1336-1340. [CrossRef]

18. Foldy, L.L. The multiple scattering of waves. I. General theory of isotropic scattering by randomly distributed scatterers. Phys. Rev. 1945, 67, 107-119. [CrossRef]

19. Lax, M. Multiple scattering of waves. Rev. Mod. Phys. 1951, 23, 287-310. [CrossRef]

20. Ya Polischuk, A. Quantum corrections to the Boltzmann equation for electrons in a disordered medium. J. Phys. B At. Mol. Phys. 1983, 16, 3845-3851. [CrossRef]

21. Borghesani, A.F.; Bruschi, L.; Santini, M.; Torzo, G. Electron mobility in neon at high densities. Phys. Rev. A 1988, 37, 4828-4835. [CrossRef] 
22. Bartels, A. Density dependence of the electron drift velocity in argon. Phys. Lett. A 1973, 44, 403-404. [CrossRef]

23. Borghesani, A.F.; Santini, M.; Lamp, P. Excess electron mobility in high-density argon gas. Phys. Rev. A 1992, 46, 7902-7909. [CrossRef]

24. Borghesani, A.F.; Santini, M. Electron localization-delocalization transition in high-density neon gas. Phys. Rev. A 1992, 45, 8803-8810. [CrossRef]

25. Ioffe, A.F.; Regel, A.R. Non-crystalline, amorphous and liquid electronic semiconductors. In Progress in Semiconductors; Gibson, A.F., Ed.; Wiley: Hoboken, NJ, USA, 1960; Volume 4, pp. 237-291.

26. Huxley, L.G.; Crompton, R.W. The Diffusion and Drift of Electrons in Gases; Wiley: New York, NY, USA, 1974.

27. Springett, B.E.; Jortner, J.; Cohen, M.H. Stability criterion for the localization of an excess electron in a nonpolar fluid. J. Chem. Phys. 1968, 48, 2720-2731. [CrossRef]

28. Wigner, E.; Seitz, F. On the constitution of metallic sodium. Phys. Rev. 1933, 43, 804-810. [CrossRef]

29. Hernandez, J.P.; Martin, L.W. Analysis of excess electron states in neon gas. Phys. Rev. A 1991, 43, 4568-4571. [CrossRef] [PubMed]

30. O'Malley, T.F. Extrapolation of electron-rare gas atom cross sections to zero energy. Phys. Rev. 1963, 130, 1020-1029. [CrossRef]

31. Lekner, J. Scattering of waves by an ensemble of fluctuating potentials. Philos. Mag. 1968, 18, 1281-1286. [CrossRef]

32. Ascarelli, G. Hall mobility of electrons in liquid xenon. J. Phys. Condens. Matter 1992, 4, 6055-6072. [CrossRef]

33. Adams, P.W.; Paalanen, M.A. Localization in a Nondegenerate Two-Dimensional Electron Gas. Phys. Rev. Lett. 1987, 58, 2106, [CrossRef] [PubMed]

34. Adams, P.W.; Paalanen, M.A. Anderson Localization of Electrons in Dense He ${ }^{4}$ Gas. Phys. Rev. Lett. 1988, 61, 451, [CrossRef] [PubMed]

35. Mott, N.F. Electrons in disordered structures. Adv. Phys. 1967, 16, 49-144. [CrossRef]

36. Mott, N. Metal-Insulator Transitions; Taylor \& Francis: Abingdon, UK, 1974.

37. Cohen, M.H.; Lekner, J. Theory of hot electrons in gases, liquids, and solids. Phys. Rev. 1967, 158, 305-309. [CrossRef]

38. Stanley, H.E. Introduction to Phase Transitions and Critical Phenomena; International Series of Monographs on Physics; Oxford University Press: Oxford, UK, 1971; Volume 46.

39. Thomas, J.E.; Schmidt, P.W. X-ray study of critical opalescence in argon. J. Chem. Phys. 1963, 39, 2506-2516. [CrossRef]

40. Borghesani, A.F. Electron mobility maximum in dense argon gas at low temperature. J. Electrostat. 2001, 53, 89-106. [CrossRef]

41. Jahnke, J.A.; Silver, M.; Hernandez, J.P. Mobility of excess electrons and O2-formation in dense fluid helium. Phys. Rev. B 1975, 12, 3420-3427. [CrossRef]

42. Borghesani, A.F.; Santini, M. High-temperature electron localization in dense He gas. Phys. Rev. E 2002, 65, 8, [CrossRef] [PubMed]

43. Borghesani, A.; Bruschi, L.; Santini, M.; Torzo, G. Density dependence of the electronic mobility in high density neon gas. Phys. Lett. A 1985, 108, 255-258. [CrossRef]

44. Borghesani, A.F.; Santini, M. Electron mobility and localization effects in high-density Ne gas. Phys. Rev. A 1990, 42, 7377-7388. [CrossRef]

45. Borghesani, A.F.; Neri, D.; Santini, M. Low-temperature $\mathrm{O}_{2}^{-}$mobility in high-density neon gas. Phys. Rev. E 1993, 48, 1379-1389. [CrossRef]

46. Neri, D.; Borghesani, A.F.; Santini, M. Electron attachment to $\mathrm{O}_{2}$ molecules in dense helium and argon gases. Phys. Rev. E 1997, 56, 2137-2142. [CrossRef]

47. Borghesani, A.F. Resonant low-energy electron attachment to $\mathrm{O}_{2}$ impurities in dense neon gas. Plasma Sources Sci. Technol. 2020, 29, 35024, [CrossRef]

48. Torzo, G. A simple recirculating pump for high-pressure high-purity gas. Rev. Sci. Instrum. 1990, 61, 1162-1163. [CrossRef]

49. Angus, S.; Reuck, K.M.D.; McCarty, R.D. International Thermodynamic Tables of the Fluid State—4 Helium; Pergamon: New York, NY, USA, 1977.

50. Sychev, V.V.; Vasserman, A.A.; Kozlov, A.D.; Spiridonov, G.A.; Tsymarny, V.A. Thermodynamic Properties of Helium; Hemisphere: Washington, DC, USA, 1987.

51. Borghesani, A.F.; Delfitto, G. A low-cost, continuously adjustable, high-voltage, regulated DC power supply. Meas. Sci. Technol. 1990, 1, 825-827. [CrossRef]

52. Borghesani, A.F.; Bruschi, L.; Santini, M.; Torzo, G. Simple photoelectronic source for swarm experiments in high-density gases. Rev. Sci. Instrum. 1986, 57, 2234-2237. [CrossRef]

53. Borghesani, A.F.; Santini, M. Electron swarm experiments in fluids-signal waveform analysis. Meas. Sci. Technol. 1990, 1, 939-947. [CrossRef]

54. Miyakawa, T.; Dexter, D.L. Stability of electronic bubbles in liquid neon and hydrogen. Phys. Rev. 1969, 184, 166-172. [CrossRef]

55. Atrazhev, V.M. Delocalisation of electrons in dense helium gas by external electric field. J. Phys. D: Appl. Phys. 1984, 17, 889-902. [CrossRef]

56. Sakai, Y.; Schmidt, W.F.; Khrapak, A. High- and low-mobility electrons in liquid neon. Chem. Phys. 1992, 164, 139-152. [CrossRef]

57. Bartels, A.K. Messungen der Elektronendriftgeschwindigkeit in He, Ar, H2 und N2 bei Sehr Hohen Gasdichten (zur Frage der kollektiven WechselWirkung von Elektronen mit dem Gas). Ph.D. Thesis, Hamburg University, Hamburg, Germany, 1974. 
58. Borghesani, A.F.; Santini, M. Electron mobility maximum in near-critical argon gas. Int. J. Thermophys. 2001, $22,1109-1121$. [CrossRef]

59. Borghesani, A.F.; Lamp, P. Injection of photoelectrons into dense argon gas. Plasma Sources Sci. Technol. 2011, 20, 34001, [CrossRef]

60. Weyhreter, M.; Barzick, B.; Mann, A.; Linder, F. Measurements of differential cross sections for e-Ar, $\mathrm{Kr}, \mathrm{Xe}$ scattering at E = $0.05-$ 2 eV. Zeitschrift Phys. D Atoms Mol. Clust. 1988, 7, 333-347. [CrossRef]

61. Borghesani, A.F. Ions and Electrons in Liquid Helium; International Series of Monographs on Physics; Oxford University Press: Oxford, UK, 2007; Volume 137.

62. McCarty, R.D.; Arp, V.D. A New Wide Range Equation of State for Helium. In Advances in Cryogenic Engineering; ACRE; Springer: Boston, MA, USA,1990; Volume 35, pp. 1465-1475. [CrossRef]

63. Haddad, G.N.; Elford, M.T. Low-energy electron scattering cross sections in carbon dioxide. J. Phys. B At. Mol. Phys. 1979, 12, [CrossRef]

64. Elford, M.; Haddad, G. The Drift Velocity of Electrons in Carbon Dioxide at Temperatures between 193 and 573 K. Aust. J. Phys. 1980, 33, 517-530. [CrossRef]

65. Krebs, P.; Giraud, V.; Wantschik, M. Electron Localization in Dense Ammonia Vapor. Phys. Rev. Lett. 1980, 44, 211, [CrossRef]

66. Krebs, P.; Lang, U. Electron Mobility and Multiple Scattering Effects in Dense Methanol Gas. J. Phys. Chem. 1996, 100, 10482-10489. [CrossRef] 